\title{
Antibiotics prescription pattern and determinants of utilization in the national health insurance scheme at a Tertiary Hospital in Nigeria
}

\author{
Roland Nnaemeka Okoro ${ }^{1}$, Chijioke Nmeka², Patrick O Erah ${ }^{3}$
}

1. Department of Clinical Pharmacy and Pharmacy Administration, Faculty of Pharmacy, University of Maiduguri, P.M.B 1069, Maiduguri, 600230, Borno State(Nigeria).

2. Department of Pharmaceutical Services, University of Nigeria Teaching Hospital, Ituku-Ozalla, Enugu (Nigeria).

3. Department of Clinical Pharmacy and Pharmacy Practice, Faculty of Pharmacy, University of Benin, Benin City (Nigeria).

\begin{abstract}
Background: Subsidizing the cost of medicines through insurance schemes increases consumption of medicines and may contribute to irrational use of antibiotics.

Objectives: To describe the systemic antibiotics prescriptions patterns and analyze the determinants of their utilization in the National Health insurance Scheme (NHIS).

Methods: Established WHO guideline was followed to conduct this cross-sectional retrospective study at University of Nigeria Teaching Hospital, Nigeria. Data were collected from randomly sampled prescription sheets of one year duration. Logistic regression analysis was performed to determine the predictors of antibiotics prescriptions.

Results: The results are based on 802 sampled out-patients NHIS prescriptions. Average number of medicines per encounter was $4.0 \pm 1.8$, whereas $46.9 \%$ of antibiotics were prescribed by generic name. Penicillins (most frequently amoxicillin/clavulanate), and nitroimidazole (most frequently metronidazole) were the most commonly prescribed antibiotics with percentage share of $43.3 \%$ and $22.2 \%$. Being $<5$ years old, and taking more than 4 medicines (OR 2.20, 95\% CI 1.37-3.55) were the factors associated with the highest risk of antibiotics exposure.

Conclusion: There were poly-pharmacy, and non-adherence to generic antibiotic prescriptions. Penicillins (amoxicillin/clavulanate) were the most commonly prescribed antibiotic class. Being $<5$ years old, and taking more than 4 medicines were significant predictors of antibiotics exposure.

Keywords: Antibiotics; national health insurance scheme; Nigeria; poly-pharmacy; prescription.

DOI: https://dx.doi.org/10.4314/ahs.v19i3.8

Cite as: Okoro RN, Nmeka C, Erah PO. Antibiotics prescription pattern and determinants of utilization in the national health insurance scheme at a Tertiary Hospital in Nigeria. Afri Health Sci. 2019;19(3): 2356-2364. https:/ / dx.doi.org/10.4314/ahs.v19i3.8
\end{abstract}

\section{Introduction}

Antibiotics are commonly used in the ambulatory and hospital outpatient settings ${ }^{1}$. The patterns of use have been shown to vary between public and private sectors ${ }^{2,3}$. Earlier studies have reported penicillins and cephalospo-

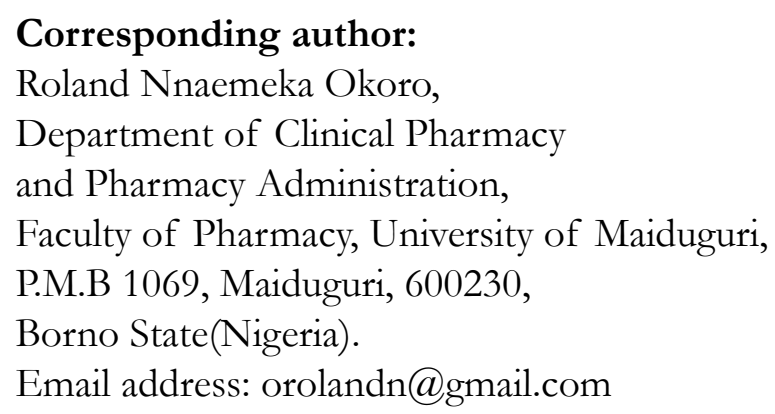

rins as the most frequently prescribed antibiotics in many clinical settings ${ }^{4-6}$. Antibiotics are powerful and effective medicines used for treating infections caused by bacteria. Also known as antimicrobial drugs, antibiotics have saved countless lives ${ }^{7}$. In spite of their effectiveness to treat many bacterial infections, they are frequently used irrationally to treat viral infections as in many cases of upper respiratory tract infections ${ }^{8}$. Generally, irrational use of medicines is common in Nigeria, resulting in poly-pharmacy, overuse of antibiotics and non-adherence to the principles of essential drug policy ${ }^{9,10}$.

In addition, subsidizing the cost of medicines through health insurance schemes increases consumption of medicines especially antibiotics, which in turn may contribute to irrational use ${ }^{11}$. The inevitable consequence of

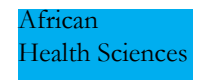

(C) 2019 Okoro et al. Licensee African Health Sciences. This is an Open Access article distributed under the terms of the Creative commons Attribution License (https://creativecommons.org/licenses/BY/4.0), which permits unrestricted use, distribution, and reproduction in any medium, provided the original work is properly cited. 
irrational use of antibiotics is the emergence of antibiotic-resistant pathogens, fueling an ever-increasing need for new drugs at a time when the pace of antimicrobial drug development has slowed dramatically ${ }^{12}$. Antimicrobial resistance is currently the greatest challenge to the effective treatment of infections globally. Antibiotic resistance not only leads to waste of health resources but also increases morbidity and mortality ${ }^{13,14}$. Therefore, appropriate use of antibiotics is an essential ingredient in the provision of quality health care, patient safety, and the rational use of health resources ${ }^{15}$ especially in a resource constrained nation like Nigeria. This underscores the need to monitoring antibiotics use in the scheme where the tendency for overuse is higher to ensure rational prescribing and use among health care providers and beneficiaries respectively.

In order to advise on effective strategies for more rational prescribing of antibiotics, a better understanding of factors with higher likelihood for an individual to have an antibiotic prescription is essential and development of targeted intervention programmes aimed at optimizing their use in those conditions is warranted.

Similar studies done under NHIS in the North East, South West, and North West Nigeria had reported irrational antibiotics use $e^{9,16-18}$. The current situation in the University of Nigeria Teaching Hospital (UNTH) with respect to prescribing and use of antibiotics under NHIS is not documented. To our knowledge, no such study has been carried out purposively in an outpatient NHIS setting of the UNTH. Therefore, this study was undertaken to provide insights into antibiotics prescribing practices of the study setting and to gather baseline information that could serve as basis for designing appropriate interventions \& policies to improve antibiotics prescribing in the scheme. The objectives of this study were to describe the systemic antibiotics prescription patterns and analyze the determinants of their utilization in the NHIS.

\section{Methods \\ Setting}

This study was conducted at the NHIS dispensing pharmacy outlet of the UNTH, Ituku-Ozalla, Enugu, South East Nigeria. UNTH is a tertiary health care facility of about 500 bed capacity. It serves as the Teaching Hospital for the Faculty of Medicine of the University of Nigeria and is a participating health care provider in the scheme, and has over 20,000 enrollees. The department of pharmaceutical services is a service arm of the hospital. It provides pharmaceutical services to both the in-patients and out-patients that visit the hospital. NHIS dispensing pharmacy outlet is a dedicated pharmacy that attends to the medicine and medicine related needs of insured patients. It opens to patients throughout the workdays but the peak hours for out-patients transactions is from 10.00 am to $1.00 \mathrm{pm}$. All NHIS essential medicines are accessed from this pharmacy outlet except injections, creams, ophthalmic drugs, ear preparations, and surgical items which are accessed from other dispensing pharmacy outlets under NHIS coverage. NHIS prescriptions are filled, kept and stored in this pharmacy outlet for a minimum period of one year before disposal. As at time of this study, the average number of prescriptions dispensed per day was 50, and 4 pharmacists, 2 intern Pharmacists, 1 pharmacy technician, 1 pharmacy assistant, and 1 clerk were working in this pharmacy outlet.

\section{Study design}

This was descriptive, cross-sectional retrospective study that utilized prescription sheets issued to out-patients seen and treated under NHIS at the health care facility between January and December 2013.

\section{Sampling}

Systematic sampling with an interval of 20 was used to select prescriptions that were included in the study and simple balloting was used for the first pick.

\section{Ethical consideration}

Ethical approval was obtained from the Research and Ethics Committee of the hospital before the commencement of data collection.

\section{Data collection}

The data for this study were collected between August-October 2014 from the NHIS out-patient's prescription sheets of twelve months period of 2013.The following information including patient age in years, gender, months of prescription, number of all medicines prescribed, number of antibiotics prescribed, number of NHIS essential antibiotics prescribed, number of NHIS essential antibiotics prescribed by generic name, number of NHIS essential antibiotics dispensed, name of individual antibiotics and therapeutic class were extracted 
from the sampled prescription sheets into the pretested data collection forms.

\section{Data analysis}

The WHO drug use indicators investigated in this study were average number of medicines per encounter, average number of antibiotics per encounter, percentage of antibiotics prescribed by generic name, percentage of antibiotics prescribed from NHIS essential medicine list (EML) 2005 edition, and percentage of essential antibiotics dispensed ${ }^{15,19}$. These indicators were specifically chosen because they would provide information on particular areas of interest to the investigators.

The values of the prescribing indicators were calculated as follows:

1. Average number of drugs prescribed per encounter $=$ total number of drugs prescribed $\div$ total number of encounters surveyed

2. Average number of antibiotics prescribed per encounter $=$ total number of antibiotics prescribed $\div$ total number of encounters surveyed

3. Percentage of NHIS essential antibiotics prescribed by generic name $=$ (number of NHIS essential antibiotics prescribed by generic name $\div$ total number of NHIS es- sential antibiotics prescribed) X 100

4. Percentage of antibiotics prescribed from NHIS EML $=$ (number of antibiotics prescribed from EML $\div$ total number of antibiotics prescribed) X 100.

5. Percentage of NHIS essential antibiotics dispensed $=$ (number of NHIS essential antibiotics dispensed $\div$ total number of NHIS essential antibiotics prescribed) X 100 Statistical analysis was done using the Statistical Package for Social Sciences (SPSS) version 21 (SPSS inc. Chicago) for Windows ${ }^{\circledR}$ and Microsoft ${ }^{\circledR} 2007$ Excel package. The results were presented as mean (standard deviation), range, frequencies and percentages. Logistic regression analysis was performed to determine the predictors of antibiotics exposure. Statistical significance was set at $\mathrm{p}$ $<0.05$.

\section{Results}

A total of 802 prescriptions were successfully analyzed. All encounters had 3214 drugs with an average of 4 medicines per prescription ranging from 1-11 medicines. The proportion of prescriptions with more than 4 medicines was $36.4 \%$. Of all the medicines prescribed, 275 were antibiotics and $46.9 \%$ of these antibiotics were prescribed by generic name, whereas $82.9 \%$ were prescribed from NHIS EML as shown in Table 1.

Table 1: Antibiotics prescribing indicators

\begin{tabular}{ll}
\hline Medicine Prescribing Indicator & Value \\
\hline Average number of medicines per prescription (Mean \pm SD) & $4.0 \pm 1.8$ \\
Average number of antibiotics per encounter(Mean \pm SD) & $1.3 \pm 0.5$ \\
Percentage of antibiotics prescribed by generic name (\%) & 46.9 \\
Percentage of antibiotics prescribed from NHIS EML (\%) & 82.9 \\
Percentage of antibiotics dispensed (\%) & 72.4 \\
\hline SD = Standard Deviation
\end{tabular}

$S D=$ Standard Deviation

A total of 211 encounters contained at least an antibiotic. Majority of patients that were prescribed antibiotics were females $(57.3 \%)$ The mean age of patients exposed to antibiotics was $33.9 \pm 19.3$ years with majority within the age group of $12-59$ years $(72.5 \%)$. Most of the antibiotics were prescribed in the second quarter of the year $(34.6 \%)$ as shown in Table 2. 
Table 2: Distribution of prescribed antibiotics in various age groups, gender and period of the year

\begin{tabular}{llr}
\hline Variable & & Antibiotics Prescribed $\mathbf{n}(\%)$ \\
\hline Age Group (Years) & $<5$ & $20(9.5)$ \\
& $5-11$ & $25(11.8)$ \\
& $12-59$ & $153(72.5)$ \\
Gender & $\geq 60$ & $13(6.2)$ \\
& Female & $121(57.3)$ \\
Period of the Year & Male & $90(42.7)$ \\
& $1^{\text {st }}$ Quarter & $65(30.8)$ \\
& $2^{\text {nd }}$ Quarter & $73(34.6)$ \\
& $3^{\text {rd }}$ Quarter & $62(29.4)$ \\
& $4^{\text {th }}$ Quarter & $11(5.2)$ \\
\hline
\end{tabular}

Table 3 shows the prescribing rate for each individual antibiotic. Penicillins (43.3\%), nitroimidazoles (22.2\%) followed by floroquninolones $(20.0 \%)$ were the most commonly prescribed antibiotic classes. Amoxicillin/ clavulanate, and metronidazoles were the most frequently prescribed in the penicillins, and nitroimidazoles $(73.1 \%$ vs $78.7 \%$ ) therapeutic class categories respectively. Conversely, the tetracyclines, sulphonamides, and the lincosamides were rarely prescribed, accounting for only $4.0 \%$ of the total antibiotics surveyed.

Table 3: Overall antibiotics prescription patterns $(\mathrm{N}=\mathbf{2 7 5})$

\begin{tabular}{lll}
\hline & & \\
Antibiotic Class n (\%) & Antibiotic Name & $\mathbf{n}(\%)$ \\
\hline Penicillins 119(43.3) & Amoxicillin+Clavulanate & $87(31.6)$ \\
& Amoxicillin & $21(7.6)$ \\
& Ampicillin+Cloxacillin & $7(2.5)$ \\
& Flucloxacillin & $3(1.1)$ \\
& Ampicillin+Sulbactam & $1(0.4)$ \\
Nitroimidazoles 61(22.2) & Metronidazole & $48(17.5)$ \\
& Secinidazole & $10(3.6)$ \\
Floroquinolones 55(20.0) & Tinidazole & $3(1.1)$ \\
& Ciprofloxacin & $36(13.1)$ \\
Macrolides 14(5.1) & Oevofloxacin & $14(5.1)$ \\
& Efloxacin & $5(1.8)$ \\
Cephalosporins 14(5.1) & Azithromycin & $6(2.2)$ \\
& Clarithromycin & $5(1.8)$ \\
& Cefixime & $3(1.1)$ \\
Tetracyclines 5(1.8) & Cefuroxime & $6(2.2)$ \\
& Cephalexin & $4(1.4)$ \\
Sulphonamide 5(1.8) & Doxycycline & $3(1.1)$ \\
Lincosamide 1(0.4) & Tetracycline & $1(0.4)$ \\
\hline & Cotrimoxazole & $4(1.4)$ \\
& Clindamycin & $1(0.4)$ \\
& & $5(1.8)$ \\
\end{tabular}


Table 4 shows that out of the surveyed antibiotic prescriptions, $70.5 \%$ contained one antibiotic, $28.4 \%$ contained two antibiotics, while $1.0 \%$ contained three antibiotics. Amoxicillin/clavulanate contributed the highest share (43.0\%) in the monotherapy group, Amoxicillin/ clavulanate plus metronidazole $(33.7 \%)$ was the most prescribed in the dual antibiotics combination category.

Table 5 summarized the results of the antibiotic utiliza- tion determinants analysis. Age of the patients, and number of medicines per patient encounter were the main determinants of antibiotics exposure. In particular, being between 12-59 years old (OR 0.44, 95\% CI 0.24 - 0.83), and $\geq 60$ years old (OR $0.21,95 \%$ CI 0.09 - 0.50) were significantly associated with least risk of antibiotics exposure, whereas taking more than 4 medicines was significantly associated with the highest risk of antibiotics exposure (OR 2.20, 95\% CI 1.37 - 3.55).

Table 4: Prescribed antibiotics mono- and combination therapies

\begin{tabular}{lll}
\hline Variable & Antibiotics & $\mathbf{n ~ ( \% )}$ \\
\hline Monotherapy $\mathbf{N}=\mathbf{1 4 9}$ & Amoxicillin/Clavulanate & $64(43.0)$ \\
& Ciprofloxacin & $24(16.1)$ \\
& Amoxicillin & $13(8.7)$ \\
& Ampicillin/Cloxacillin & $6(4.0)$ \\
& Erythromycin & $6(4.0)$ \\
& Cefixime & $5(3.4)$ \\
& Levofloxacin & $5(3.4)$ \\
Dual therapy $\mathbf{N}=\mathbf{6 0}$ & Others & $26(17.4)$ \\
& Amoxicillin/Clavulanate+Metronidazole & $19(31.8)$ \\
& Ciprofloxacin+Metronidazole & $11(18.3)$ \\
& Amoxicillin+Metronidazole & $7(11.7)$ \\
& Levofloxacin + Secinidazole & $6(10.0)$ \\
& Amoxicillin/Clavulanate+ Secinidazole & $2(3.3)$ \\
& Cefpodoxime+Metronidazole & $2(3.3)$ \\
& Doxycycline+Metronidazole & $2(3.3)$ \\
Triple therapy $\mathbf{N}=\mathbf{2}$ & Others & $11(18.3)$ \\
\hline & Doxycycline+Metronidazole+Levofloxacin & $2(100.0)$ \\
\hline
\end{tabular}

Table 5: Determinants of antibiotic exposure

\begin{tabular}{llll}
\hline Variable & & OR $(95 \% \mathrm{Cl})$ & P value \\
\hline Age group(years) & $<5$ & Reference & \\
& $5-11$ & $0.70(0.32-1.50)$ & 0.350 \\
& $12-59$ & $0.44(0.24-0.83)$ & $0.006^{*}$ \\
Gender & $\geq 60$ & $0.21(0.09-0.50)$ & $0.001^{*}$ \\
& Women & Reference & \\
Period of year & Men & $1.26(0.92-1.74)$ & 0.152 \\
& 1st Quarter $^{\text {2t }}$ & Reference & \\
& $2^{\text {nd }}$ Quarter & $0.79(0.54-1.17)$ & 0.237 \\
Number of & $3^{\text {rd }}$ Quarter & $0.92(0.61-1.39)$ & 0.690 \\
Medicines per & $4^{\text {th }}$ Quarter & $1.16(0.54-2.50)$ & 0.711 \\
Encounter & $1-2$ & Reference & \\
& & & \\
& & & \\
& $3-4$ & $1.63(1.02-2.62)$ & $0.042^{*}$ \\
& $\geq 5$ & $2.20(1.37-3.55)$ & $0.001^{*}$ \\
\hline
\end{tabular}

${ }^{*}$ Significant at $P<0.05$ 


\section{Discussion}

This retrospective study shows high average number of medicines per prescription and low generic antibiotic prescribing, while more than two-third of the antibiotics surveyed were prescribed from NHIS EML. Majority of patients prescribed antibiotics were within the age group of 12-59 years; while more than one-half of the total prescriptions were written for females. The highest antibiotics prescription rate was recorded in the second quarter of the year. Penicillins followed by nitroimidazoles were the most prescribed antibiotic therapeutic classes, whereas amoxicillin/clavulanate followed by metronidazole was the most prescribed individual antibiotics. More than two-third of all antibiotics encounters constituted monotherapy. Age less than 5 years old and prescribing more medicines significantly increased the probability of receiving an antibiotics prescription.

The mean number of prescribed medicines found in this study was 4.1. This result is similar to 4.1 and 3.8 reported by studies that were conducted in the NHIS of tertiary healthcare facilities in the South West, and North West Nigeria respectively ${ }^{17,18}$. In contrast, a comparison of drug prescribing practices during a pre- and post-insurance era in a Nigerian hospital found the mean prescribed drugs to be 3.0 and 2.6, respectively ${ }^{16}$. Another study conducted in the NHIS of a tertiary healthcare facility in the North East Nigeria recorded a value of 3.49. The difference between the value reported by our study and these previous studies could be due to geographical variations, differences in prescribing habits and study settings. However, a study conducted under NHIS in Ghana reported a much lower value of $2.9^{20}$. This lower value could be due to engagement of relevant stake holders on rationale prescribing habit in Ghanaian Health Insurance Scheme. Thus, the high average number of medicines per prescription in our study suggests poly-pharmacy. Poly-pharmacy increases the risk off unwanted side effects and of dangerous interactions between different medicines. The proportion of prescriptions that had more than 4 medicines was $36.4 \%$. The level of poly-pharmacy in this study is comparable to $32.4 \%$ reported in the South West Nigeria17, but contrary to $14.1 \%$ recorded in North Central Nigeria ${ }^{21}, 55.6 \%$ in Ghana ${ }^{22}$, and $47.9 \%$ in Cameroon $^{23}$ respectively.

The percentage of generic antibiotics prescribed was $46.9 \%$, which is considerably lower than the standard of
$100 \%$. This finding represents a severe underuse of generic antibiotics in the study setting. This result is consistent with the range of $45.8 \%-57.0 \%$ reported by previous studies on general NHIS prescription audits ${ }^{9,16-18}$ and non-NHIS prescription audits conducted in different parts of Nigeria ${ }^{21,24}$ and health insurance studies in Sudan that found $54.2 \%$ and $38.5 \%$ respectively ${ }^{25,26}$. When compared with the result of an earlier study done in another West African country, our value was far lower than $99.8 \%$ found in the NHIS in Ghana ${ }^{20}$. These much lower values were reported in Nigerian NHIS despite the fact that NHIS was established on generic medicine policy, in order to reduce health care cost on medicines. Therefore, these findings in Nigerian NHIS suggest poor implementation of this policy by relevant stakeholders. The implications of low generic use are primarily the wastage of scarce health resources and a decrease in access to pharmaceuticals because of an affordability barrier.

The concept of essential medicines was introduced to help streamline the number of medicines deployed within the health care system in order to improve rational prescribing and reducing health care costs. In this current study, $82.9 \%$ of all prescribed antibiotics were from the Nigerian NHIS EML. This is close to $91.0 \%$ reported by a study on general NHIS prescription audits in the North West Nigeria ${ }^{18}$. An NHIS study done in Sudan found $99.3 \%$ adherence to $\mathrm{EML}^{25}$. These findings indicate that there is still enough room for improvement in order to attain the $100 \%$ benchmark in our study setting. A study conducted in Ghana demonstrated that it is feasible to achieve an absolute adherence (100\%) to EML in health insurance scheme ${ }^{20}$. The reason for this full adherence is because the Ghanaian insurance scheme reimburses only medicines prescribed from NHIS EML. Therefore, Nigerian NHIS should take cue from its Ghanaian counterpart and restrict medicines reimbursement to only medicines contained in the scheme's EML.

The current study showed higher antibiotic prescriptions for females than males consistent with the finding of a previous study done in Ghana ${ }^{20}$. Patients aged between 12-59 years had the highest antibiotic prescriptions when compared to other age groups and most of these antibiotics were prescribed in the second quarter of the year. This result is incongruent with the Ghanaian study that reported highest antibiotic prescriptionsfor patients below 5 years and in the first quarter of the year respective$1 y^{20}$. 
Our study revealed that penicillins followed by nitroimidazoles were the most prescribed antibiotic classes. This pattern is similar to the report of earlier study conducted in North West part of the country ${ }^{27}$, but contrary to the findings of other similar Nigerian studies ${ }^{21,28,29}$, and study done in a neighboring Cameroon23. Amoxicillin/clavulanate followed by metronidazole was the most frequently prescribed individual antibiotics comparable to amoxicillin, and metronidazole reported by similar non-insurance studies ${ }^{27,29}$. This result is not in agreement with the findings of non-insurance studies done in other parts of the country $y^{28,30,31}$ and Ghana ${ }^{22}$. The preference of Amoxicillin/clavulanate over other most prescribed antibiotics could be increasing affordability due to health insurance coverage.

Overall, $70.6 \%$ patients were treated with antibiotic monotherapy comparable with $96.5 \%$ reported by an Indian study ${ }^{32}$, but contrary to $38.1 \%$ found by a similar study conducted in the South East Nigeria31 and 39.5\% reported by an Ethiopian study33. The antibiotic combination pattern showed that a combination of amoxicillin/ clavulanate plus metronidazole $(31.7 \%)$ had the highest share among the dual therapy prescriptions comparable with other studies that reported amoxicillin and metronidazole in the North West and South South Nigeria ${ }^{27-29}$, but contrary to ciprofloxacin plus metronidazole recorded in Ghana ${ }^{22}$. Levofloxacin plus metronidazole plus doxycycline were the only triple combination recorded in the current study contrary to ciprofloxacin plus metronidazole plus doxycycline, and amoxicillin plus metronidazole plus doxycycline $(21.5 \%)$ reported in North West Nigeria ${ }^{27,28}$. The differences in the observed prescribing patterns might be due to their availability in the hospital, differences in pathogen characteristics, physicians' antibiotic prescribing practices, antibiotic demand and patient characteristics, cultural and socioeconomic factors, and healthcare and legal environment.

Age groups 12-59 years and 60 years and above have lower risk of antibiotic exposure. This result is similar to the finding of a study conducted in Ghana ${ }^{20}$. This finding could be due to high prevalence of acute diarrhoeal disease and respiratory tract infections among children under 5 years in Nigeria $^{34}$. Studies done in Nigeria con- firmed that the exposure to antibiotics in children under 5 years of age is exceptionally high ${ }^{35}$. Studies from Portugal, Jordan, and Ethiopia also reported very high antibiotics exposure in children under 5 and fewer than 6 years of age ${ }^{36-38}$. Furthermore, this study revealed that increasing the number of medicines per encounter also increased the risk of receiving an antibiotic prescription. This finding suggests that antibiotics contribute significantly to poly-pharmacy recorded in this study.

\section{Limitations}

Only prescribed antibiotics were studied, drug prescription does not guarantee actual use. Secondly, the findings apply to only NHIS at the UNTH, and may thus be not representative of other NHIS settings in Nigeria which restricts generalization of the findings. Lastly, the study findings may not reflect the current antibiotics prescribing practice of the setting studied due to time lag between data collection and publishing of findings.

\section{Conclusion}

There were poly-pharmacy and non-adherence to generic antibiotic prescribing, whereas prescribing antibiotics from the essential medicine list was commendable but fell below the $100 \%$ of the NHIS guideline. Penicillins followed by nitroimidazoles were the most commonly prescribed antibiotic classes, whereas amoxicillin/clavulanate, and metronidazole were the most predominately prescribed individual antibiotics. Amoxicillin/clavulanate plus metronidazole was the most commonly prescribed antibiotic combination. Age, and number of medicines per encounter were significant determinants of antibiotic exposure.

\section{Funding}

This research did not receive any specific grant from funding agencies in the public, commercial, or not-forprofit sectors.

\section{Acknowledgement}

The authors would like to extend their gratitude to Pharm (Dr.) Justin Ogbonna, Dr. Ndubuisi Ezema and staffers at the NHIS dispensing pharmacy outlet of UNTH for their technical support during data collection processes. 


\section{Conflict of interest}

Authors declared no conflict of interest.

\section{References}

1. Boucher M, Vaillancourt R, McCarthy A. Drug use evaluation of oral antibiotics prescribed in the ambulatory care settings in the Canadian armed forces. Can J Clin Pharmacol 2003; 10: 5-10.

2. Mohlala G, Peltzer K, Phaswana-Mafuya N, Ramlagan S. Drug prescription habits in public and private health facilities in 2 provinces in South Africa. East Mediterr Health J 2010; 16: 324328.

3. Siddiqi S, Hamid S, Rafique G, Chaudhry SA, Ali N, Shahab S, Sauerborn R. Prescription practices of public and private health care providers in Attock District of Pakistan. Int J Health Plann Manage 2002; 17: 23-40.

4. Pathak A, Mahadik K, Dhaneria SP, Sharma A, Erikson B, Lundborg CS. Antibiotic prescribing in outpatients: Hospital and seasonal variations in Ujjain, India. ScandJ Infect Dis 2011.

5. Sawalha AF, Sweileh WM, Zyoud SH, Al-Jabi SW, Shamseh FF, Odah A. Analysis of prescriptions dispensed at community pharmacies in Nablus, Palestine. East Mediterr Health J 2010; 16: 788792.

6. Sahin H, Arsu G, Köseli D, Büke C. Evaluation of primary health care physicians' knowledge on rational antibiotic use. Mikrobiyol Bul 2008; 42: 343-348.

7. FDA. Combating Antibiotic Resistance. U.S Food and Drug Administration, 2011. Available from: https:// www.fda.gov/ForConsumers / ConsumerUpdates / ucm092810.htm. (Accessed September 04, 2017).

8. A, Hurst C, Hou X. Antibiotics Overuse in Children with Upper Respiratory Tract Infections in Saudi Arabia: Risk Factors and Potential Interventions. Clinical Medicine and Diagnostics. 2011; 1: 8-16.

9. Okoro RN, Shekari BG. Physicians' drug prescribing patterns at the National Health Insurance Scheme unit of a teaching hospital in the North Eastern Nigeria. Arch Pharm Pract. 2013; 4: 3-8

10. Enato EFO, Chima IE. Evaluation of drug utilization patterns and patient care practices. West Afri J Pharm 2011; 22: 36-41.

11. Faden L, Vialle-Valentin C, Ross-Degnan D, Wagner A. The role of health insurance in the cost-effective use of medicines. WHO/HAI Review Series on Pharma- ceutical Pricing Policies and Interventions. Working paper 2014; 2: 1-4 PubMed .

12. Brunton L, Parker K, Blumenthal D, Buxton L. Chemotherapy of Microbial Diseases. Goodman and Gilman's manual of pharmacology and therapeutics. $11^{\text {th }}$ Edition. The McGraw Hill companies USA, 2008: 707-711. 13. Leung E, Weil DE, Raviglione M, Nakatani H. World Health Organization World Health Day Antimicrobial Resistance Technical Working Group. The WHO policy package to combat antimicrobial resistance. Bull World Health Organ 2011; 89: 390-392.

14. Ganguly NK, Arora NK, Chandy SJ, Global Antibiotic Resistance Partnership (GARP)-India Working Group, et al. Rationalizing antibiotic use to limit antibiotic resistance in India. Indian J Med Res 2011;134: 281-294.

15. Anker M, Jokobowicz B, Fresle DA, Hozerzil HV. How to investigate drug use in health facilities. WHO/ DAP, 1993.

16. Adebayo ET, Hussain NA, Ajanaku VS. Influence of health insurance on rational use of drugs. TAF Prev Med Bull 2013; 12: 511-518.

17. Fadare JO, Adeoti AO, Aina F, Solomon OA, Ijalana JO. The influence of health insurance scheme on the drug prescribing pattern in a Nigerian tertiary healthcare facility. Niger Med J 2015a; 56: 344-348.

18. Abdu-Aguye SN, Haruna A, Shehu A, Labaran KS. An assessment of antimicrobial prescribing at a tertiary hospital in north-western Nigeria. AfrJ Pharmacol Ther 2016; 5: 229-234.

19. NHIS. Healthcare Providers Service Price List. 2005. Available from: http://dhmlnigeria.com/downloads/ NHIS_drugs_pricelist_2011.pdf.(Accessed September 4, 2017).

20. Apanga S, Chirawurah D, Kudiabor C, Adda J, Adoesom JA, Punguyire D. Evaluation of Drug Prescribing Pattern under the National Health Insurance Scheme in Rural Ghana. IJPSR 2014; 5: 21932198.

21. Akande TM, Ologe M, Medubi, GF. Antibiotic prescription pattern and cost at University of Ilorin Teaching Hospital, Ilorin, Nigeria. International Journal of Tropical Medicine 2009; 4: 50-54.

22. Boadu EA. The Use of Antibiotics and the Pattern of Antimicrobial Resistance at S.D.A Hospital, Kumasi, Ghana. [Master's thesis]. Kwame Nkrumah University of Science and Technology, 2014 
23. Mbam LA, Monekosso GL, Asongalem EA.. Indications and Patterns of Antibiotic Prescription in the Buea Regional Hospital of Cameroon. Health Sci Dis 2015; 16: $1-7$.

24. Ogbonna, B., Eze, L., 2016. Antibiotic Drugs Utilization Pattern in Two Tertiary Health Facilities in South East Nigeria: Implication for Bacterial Treatment. Value in Health 2015; 19. [Abstra $\mathrm{ct}]$.

25. Mustafa IEMA. The pattern of medicines use in Sudan: a cross sectional study at National Health Insurance Fund setting, in 2012. Swiss Tropical and Public Health Institute. [Master's Thesis]. University of Basel, 2013. Available from: http://apps.who.int/medicinedocs/ en/d/Js20953en/ (Accessed September 05, 2018). Yousif BME, Supakankunti S. General Practitioners' Prescribing Patterns at Primary Healthcare Centers in National Health Insurance, Gezira, Sudan. Drugs -Real World Outcomes 2016; 3: 327332.

26. Jimoh AO, Omar I, Adebisi IM, Sani Z, Bello A, et al. Review of Morbidity Profiles and Drug Prescribing Patterns of a University Clinic in North-Western Nigeria. Int J Med Sci 2014; 1: 107115.

27. Jimoh AO, Etuk EU, Sani Z, Shuaibu HA. The Pattern of Antibiotic Use in a Family Medicine Department of a Tertiary Hospital in Sokoto, North Western Nigeria. JCDR 2011; 5: 566569.

28. Eshiet UI, Effiong GS, Akwaowoh AE. The Use of Antibiotics in a Nigerian Tertiary Health Care Facility. AJBSE 2015; 3: 2531.

29. Enato EFO, Uwaga CF. Profile of Antimicrobial Drug Use Patterns in a Nigerian Metropolitan City. Int J Health Res 2011; 4:37-44.
30. Nsofor CA, Amadi ES, Ukwandu NCD, Obijuru CE, Ohalete CV. Prevalence of Antimicrobial Use in Major Hospitals in Owerri, Nigeria. EC Microbiology 2016; 3: 522527.

31. D, Pandey K, Sharma RK. A study on drug utilization pattern of antimicrobials in outpatient departmentof medicine at tertiary care hospital. Int J Res Pharm Sci 2014; 4: 4045.

32. Woldu MA, Suleman S, Workneh N, Berhane H. Retrospective Study of the Pattern of Antibiotic Use in Hawassa University Referral Hospital Pediatric Ward, Southern Ethiopia. J App Pharm Sci 2013; 3: 93-98.

Child Care. Five Most Common Diseases Affecting Children Under 5 Years. Nigerian Bulletin, 2015. Available from: https://www.nigerianbulletin.com/threads/ child-care-5-most-common-diseases-affecting-childrenunder-5-years.113902/.(Accessed September 03, 2017)

33. Fadare J, Olatunya O, Oluwayemi O, Ogundare O. Drug Prescribing Pattern for Under-Fives in a Paediatric Clinic In South-Western Nigeria. EthiopJ Health Sci2015b; 25: 7378.

34. Al-Niemat SI, Aljbouri TM, Goussous LG, Efaishat RA, Salah RK. Antibiotic Prescribing Patterns in Outpatient Emergency Clinics at Queen Rania Al Abdullah II Children's Hospital, Jordan. Oman Medical Journal 2014; 29: 250-254.

35. Cole CP, James PB, Kargbo AT. An evaluation of the prescribing patterns for under-five patients at a Tertiary Paediatric Hospital in Sierra Leone.J Basic Clin Pharma 2015; 6:109-114.

36. Machado HS, Marafona AS, Meireles JP, Nunes CS, Esteves AL, et al. The Incidence of Exposure to Antibiotics in Children Less Than 6 Years of Age: A Survey in a Portuguese Metropolitan Area. J Preg Child Health 2016; 3: 292. 